Gastroenterologe 2016 $11: 479-488$

DOI 10.1007/s11377-016-0114-4

Online publiziert: 20. Oktober 2016

(c) Der/die Autor(en) 2016. Dieser Artikel ist eine Open-Access-Publikation.

\section{Redaktion}

R.M. Schmid, München

C. Trautwein, Aachen

CrossMark

\author{
T. Luedde ${ }^{1,2} \cdot$ C. Roderburg ${ }^{1} \cdot$ M. Binnebösel ${ }^{3} \cdot$ U. P. Neumann ${ }^{3} \cdot$ C. Trautwein \\ ${ }^{1}$ Abteilung für Gastroenterologie, Stoffwechselerkrankungen und Internistische Intensivmedizin \\ (Medizinische Klinik III), Universitätsklinikum RWTH Aachen, Aachen, Deutschland \\ ${ }^{2}$ Mildred-Scheel-Professor für Gastroenterologie, Hepatologie und hepatobiliäre Onkologie, \\ Universitätsklinikum RWTH Aachen, Aachen, Deutschland \\ ${ }^{3}$ Klinik für Allgemein-, Viszeral- und Transplantationschirurgie, Universitätsklinikum RWTH Aachen, \\ Aachen, Deutschland
}

\title{
Interdisziplinäre Therapie kolorektaler Lebermetastasen
}

enten mit kolorektalen Lebermetastasen (KLM) zwischen 12 und 15 Monaten und das 5-Jahres-Überleben unter $5 \%$ liegen [3], konnte in den letzten Jahren und Jahrzehnten durch neue und intensivere Therapieformen das mittlere Überleben der Patienten deutlich gesteigert werden. Grundsätzlich stehen heute verschiedene Therapiemodalitäten zur Verfügung (• Abb. 1). So ist die Resektion primär resektabler Lebermetastasen ein Standardvorgehen und erreicht 5-Jahres-Überlebensraten zwischen 21 und $51,8 \%$ und 10-Jahres-Überlebenszahlen (und damit Heilungsraten) von $12-36,6 \%$ [4]. In einer interdisziplinären Beratung gilt es zu entscheiden, ob Patienten mit primär resektablen Lebermetastasen zusätzlich zur Operation einer perioperativen oder adjuvanten Chemotherapie zugeführt werden. Primär nichtresektable Patienten können wiederum einer sog. Konversionschemotherapie zugeführt werden mit dem bereits initial formulierten Ziel, eine sekundäre Resektabilität zu erreichen. Aber auch Patienten, die aufgrund ihrer initialen Tumorlast und Verteilung nicht für eine Metastasenresektion vorgesehen sind, sollten im Verlauf ihrer Behandlung bei gutem Ansprechen interdisziplinär besprochen werden, um rechtzeitig zu evaluieren, ob durch Resektion doch noch eine Tumorfreiheit oder aber eine Chemotherapiepause erreicht werden kann. Schließlich haben neben chirurgischen und systemischen Behandlungsverfahren zunehmend auch interventionelle ablative Techniken Ein- zug in die Therapie von Patienten mit kolorektalen Tumoren gefunden. Lokalablative Techniken umfassen z. B. die Radiofrequenzablation (RFA), Kryoablation, Mikrowellenablation, irreversible Elektroporation (IRE) sowie die stereotaktische Bestrahlung. Regional-ablativ können die Chemoembolisation (transarterielle Chemoembolisation, TACE)und Radioembolisation (selektive interne Radiotherapie, SIRT) zur Anwendung gebracht werden. Während diese Verfahren früher v. a. in späten Linien und hochpalliativen Situationen verwendet wurden, halten sie in Kombination z. B. mit Chemotherapien und Chirurgie zunehmend auch in frühen Linien Einzug in Therapiekonzepte.

\section{》) Das mittlere Überleben der Patienten konnte deutlich gesteigert werden}

Im Folgenden wird ein kurzer Überblick über wichtige Studiendaten gegeben. Sie können eine Grundlage für eine interdisziplinäre Entscheidung darstellen, welche Therapiemodalität in welcher Kombination und an welcher Stelle einer langfristig angelegten Therapie zum Einsatz kommen kann. Aus Platzgründen können weitere wichtige Therapieverfahrensbestandteile, wie Psychoonkologie, Ernährungstherapie, Palliativmedizin etc., trotz ihrer äquivalenten Wichtigkeit zu den zuvor erwähnten Modalitäten in diesem Artikel nicht besprochen werden. 


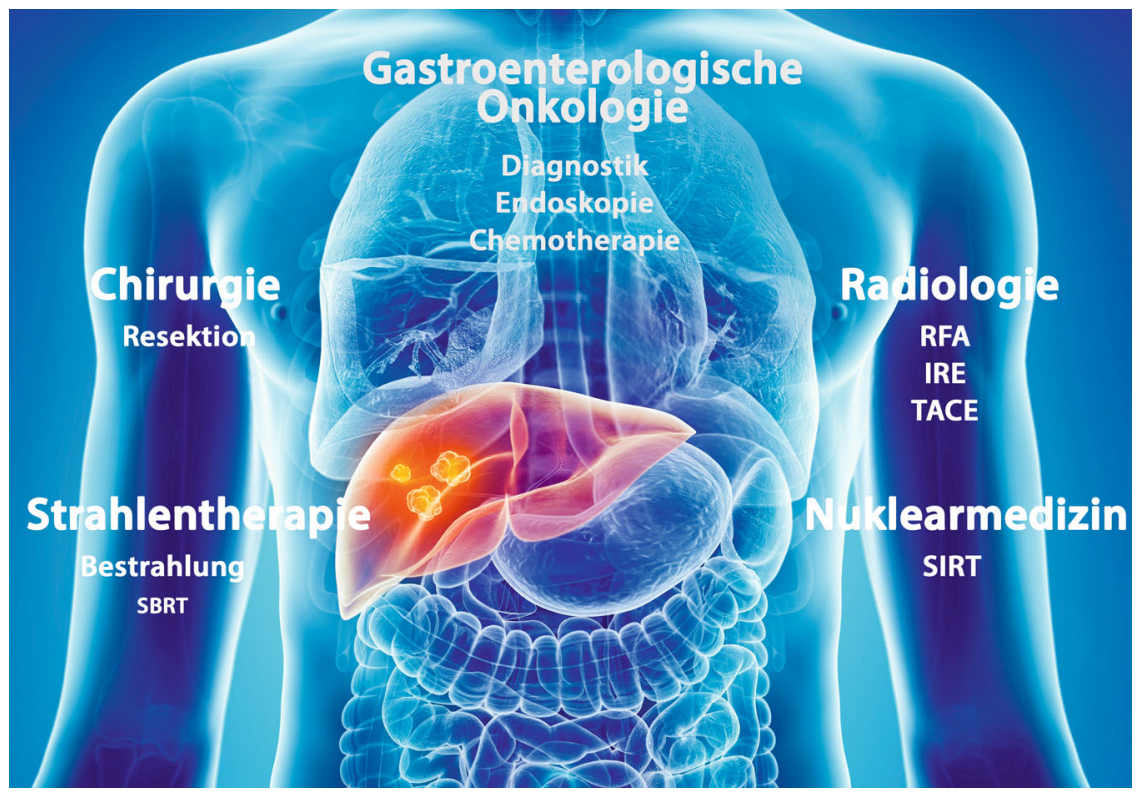

Abb. 1 A Interdisziplinäre Behandlung von Patienten mit metastasiertem Kolorektalkarzinom. IRE irreversible Elektroporation, RFA Radiofrequenzablation, SBRT "stereotactic body radiotherapy", SIRT selektive interne Radiotherapie, TACE transarterielle Chemoembolisation. CFotolia/Uniklinik RWTH Aachen

\section{Chirurgische Therapie}

Entsprechend der S3-Leitlinie zum KRK ist es vor Einleitung einer Therapie zunächst obligat, dass die metastasierten KRK-Patienten in einer interdisziplinären onkologischen Tumorkonferenz vorgestellt und die Behandlungsstrategien festgelegt werden [5]. Die Entscheidung über die Resektabilität von Lebermetastasen sollte dabei durch einen in der hepatobiliären Chirurgie erfahrenen Viszeralchirurgen erfolgen. Ebenso obligat ist die regelmäßige Vorstellung von primär als nichtresektabel eingestuften Patienten zur neuerlichen Evaluation einer potenziellen Leberresektion.

Die Indikationsstellung zur Leberresektion ist multifaktoriell. Neben der Einschätzung der reinen technischen Machbarkeit, die in hohem Maß mit der Erfahrung des Chirurgen korreliert, spielt v. a. auch eine Rolle, inwieweit eine ausgedehnte Leberresektion onkologisch sinnvoll ist. Grundsätzlich muss der Patient operationsfähig sein und es sollte eine präoperative Evaluation der Leberfunktion erfolgen. Patienten mit einer deutlich reduzierten Leberfunktion sind für eine Leberresektion in aller Regel nicht geeignet. Bei ausgedehnten Resektionen (erweiterte Hemihepatektomien) oder bei Patienten mit eingeschränkter Leberfunktion ist die präoperative Bestimmung des Lebervolumens (CTVolumetrie, - Abb. 2) und der Lebersyntheseleistung durch eine leberspezifische Funktionsbestimmung (LiMAX ${ }^{\circledR}[\mathrm{Hu}-$ medics GmbH, Marie-Elisabeth-LüdersStraße 1, D-10625 Berlin]) zu fordern [6]. Alternativ werden derzeit MRTund szintigraphiebasierte Messungen der Leberfunktion evaluiert.

Die Grundbedingung für eine kurative Leberresektion ist das Erreichen einer vollständigen hepatischen Tumorfreiheit, wobei allerdings viele Studien inzwischen auch bei R1-Resektionen sehr niedrige lokale Rezidivraten und gute langfristige Ergebnisse zeigten [7]. Ziel ist jedoch die R0-Resektion. Sie kann bei tumorfreiem Resektionsrand unabhängig vom Mindestabstand als erreicht angesehen werden, da sich unabhängig vom Resektionsabstand gleichwertige onkologische Ergebnisse zeigten [7, 8]. Zwar wird in der klinischen Praxis zur Abschätzung der Prognose der hepatisch metas-

\section{》) Patienten mit deutlich reduzierter Leberfunktion eignen sich nicht für eine Leberresektion}

tasierten KRK-Patienten der Fong-Score verwendet. Es ist unabhängig davon aber akzeptiert, dass nicht die Anzahl der Metastasen prognostisch entscheidend ist, sondern die Frage, ob alle Läsionen vollständig reseziert werden können.

Die Frage der Reihenfolge der Resektion von Primarius oder Lebermetastasen bei synchron hepatisch metastasierten KRK ist bis heute noch nicht abschließend geklärt. Vielversprechende prospektiv randomisierte Studien rekrutieren derzeit noch aktiv. Während bei Lokalisationen im rechten Kolon häufig eine simultane Resektion von Primarius und Lebermetastasen unter individueller Abwägung der perioperativen Morbidität erfolgt, setzt sich bei Rektumkarzinomen der aktuellen Literatur folgend der „liver-first approach“ durch. So zeigte sich in einer aktuellen Kohortenstudie das reverse Vorgehen mit zunächst Sanierung der Leber und erst sekundärer Resektion des Rektumkarzinoms als sicheres und effektives Verfahren mit onkologisch vielversprechenden Ergebnissen [9].

\section{》) Bei Rektumkarzinomen setzt sich der "liver-first approach" durch}

Da zur Diagnosestellung der hepatischen Metastasierung nur 10-12 \% der Patienten überhaupt einer primären chirurgischen Resektion zugänglich sind, werden unterschiedliche Therapiestrategien eingesetzt, um die Rate an potenziell resektablen Patienten zu erhöhen. Diese Strategien zielen entweder darauf ab, das Tumorvolumen zu reduzieren (neoadjuvante Chemotherapie, siehe Konversionstherapie) oder das Lebervolumen des verbleibenden Leberrests zu vergrößern (portalvenöse Embolisation oder -ligatur, 2-zeitige Leberresektionen, ,associating liver partition and portalvein ligation for staged hepatectomy“, ALPPS;

\section{- Abb. 2 und 3).}

Alternativ oder auch additiv werden zur klassischen Resektion weiterhin interventionelle Techniken eingesetzt. Patienten mit multinodulärem und bilobärem Befall oder anatomisch ungünstiger Lage der Metastase/n können mit zunehmender Tendenz mithilfe einer neo- 
Gastroenterologe 2016 11:479-488 DOI 10.1007/s11377-016-0114-4

(c) Der/die Autor(en) 2016. Dieser Artikel ist eine Open-Access-Publikation.

\section{T. Luedde $\cdot$ C. Roderburg · M. Binnebösel · U. P. Neumann · C. Trautwein}

\section{Interdisziplinäre Therapie kolorektaler Lebermetastasen}

\section{Zusammenfassung}

Hintergrund. Lebermetastasen treten bei jedem zweiten Patienten mit kolorektalem Karzinom auf.

Fragestellung. Therapiemöglichkeiten von Lebermetastasen werden vorgestellt, spezifische Indikationen dargestellt und interdisziplinärer Überlegungen aufgezeigt. Material und Methoden. Es werden die vorhandene Literatur und Leitlinienempfehlungen ausgewertet sowie Studienergebnissen und Expertenempfehlungen diskutiert.

Ergebnisse. Die chirurgische Resektion primär resektabler Lebermetastasen ist Standard und ermöglicht grundsätzlich eine Heilung in bis zu $36 \%$ der Fälle. Eine adjuvante Chemotherapie kann erwogen werden, während die Datenlage im primär resektablem Stadium bezüglich einer perioperativen Chemotherapie nicht eindeutig ist. Eine sekundäre Resektabilität primär nichtresektabler Lebermetastasen kann durch eine interventionelle Hypertrophieinduktion oder eine neoadjuvante Chemotherapie (Konversionstherapie) erreicht werden. Aktuelle Studien zeigen dabei einen möglichen Nutzen intensiverer Kombinationschemotherapien, wobei mögliche Nebenwirkungen berücksichtigt werden müssen. Schließlich spielen lokal- und regional-ablative Verfahren einen zunehmend wichtigen Teil in der multimodalen Therapie. Aktuelle Studien geben Hinweise auf einen möglichen Nutzen in Kombination mit anderen Therapien bereits in frühen Linien.

Schlussfolgerungen. Für die Therapie kolorektaler Lebermetastasen stehen verschiedene Modalitäten zur Verfügung. Die sinnvolle Koordination der einzelnen Therapieverfahren erfordert eine enge interdisziplinäre Zusammenarbeit. Patienten mit kolorektalen Lebermetastasen sollten nicht nur zu Beginn, sondern regelmäßig auch im Verlauf in einer interdisziplinären Tumorkonferenz vorgestellt werden.

Schlüsselwörter

Kolorektales Karzinom · Operation .

Chemotherapie · Adjuvante Chemotherapie $\cdot$ Interdisziplinäres Expertenteam

\section{Interdisciplinary management of colorectal liver metastases}

\section{Abstract}

Background. Liver metastases occur in every second patient with colorectal carcinoma. Objectives. Therapeutic options for patients with hepatic metastases from colorectal cancer (CRC), specific indications, and interdisciplinary concepts are presented. Methods. Based on the current literature and guidelines, novel study results and expert opinions are discussed.

Results. Surgical resection of primarily resectable liver metastases from CRC is standard and allows long-term control or healing in up to $36 \%$ of cases. Adjuvant chemotherapy after resection can be performed, but the current study data are insufficient to generally recommend perioperative chemotherapy in this setting. Secondary resectability of primarily irresectable metastases can be reached by interventional induction of liver hypertrophy or neoadjuvant chemotherapy (conversion therapy). New study results suggested a benefit for more intensive combination chemotherapies, but possible side effects have to be considered. Finally, locoregional ablative therapies have gained increasing importance in the multimodal treatment of hepatic CRC metastases, and current clinical trials suggest a possible benefit of combination strategies together with chemotherapy and surgery even in early therapy lines.

Conclusions. Liver metastases from CRC require an multidisciplinary approach. Therefore, patients should be presented to a multidisciplinary tumor board not only at the beginning, but also along different therapy lines.

Keywords

Colorectal carcinoma · Surgery · Chemotherapy - Adjuvant chemotherapy . Interdisciplinary health team adjuvanten Chemotherapie einer kurativen Leberresektion zugeführt werden. Ziel dieser Therapie ist eine möglichst rasche und weitgehende Tumorgrößenabnahme. Während die Darstellung der systemischen und auch der lokal-ablativen Therapieoptionen im Folgenden separat und ausführlich erfolgt, muss bedacht werden, dass immer auch das potenziell hepatotoxische Nebenwirkungsspektrum der modernen Chemotherapeutika berücksichtigt werden muss. Oxaliplatin beispielsweise kann zu einer sinusoidalen Obstruktion und damit zum klinischen Bild der „blue liver“ führen [10]. Irinotecan kann typischerweise eine Le- berverfettung oder eine chemotherapieassoziierte Steatohepatitis (CASH) induzieren [11]. Hepatotoxische Nebenwirkungen gehen mit einer signifikanten Erhöhung der perioperativen Morbidität und Mortalität einher und müssen daher kritisch mit abgewogen werden. Es muss jedoch auch immer bedacht werden, dass bei der Wahl des primären Regimes ein möglichst effektives Vorgehen gewählt werden sollte, da bei Versagen einer Erstlinientherapie in der Zweitlinientherapie nur noch $10 \%$ der Patienten einer Resektion zugeführt werden können $[12,13]$.
Eine weitere Strategie zur Erhöhung der potenziell resektablen Patienten stellt die präoperative Leberhypertrophieinduktion dar. Erscheint der potenziell verbleibende Leberanteil präoperativ als $\mathrm{zu}$ klein, dann ist eine radiologischinterventionelle Embolisation des zum Tumor führenden Pfortaderasts zur Hypertrophieinduktion beim tumorfreien Leberlappen die Methode der Wahl (- Abb. 2). Die portalvenöse Embolisation ist effektiv, sicher und hat eine hohe technische und klinische Erfolgsrate von $99 \%$ mit einer medianen Hypertrophie von $38 \%$ [14]. 

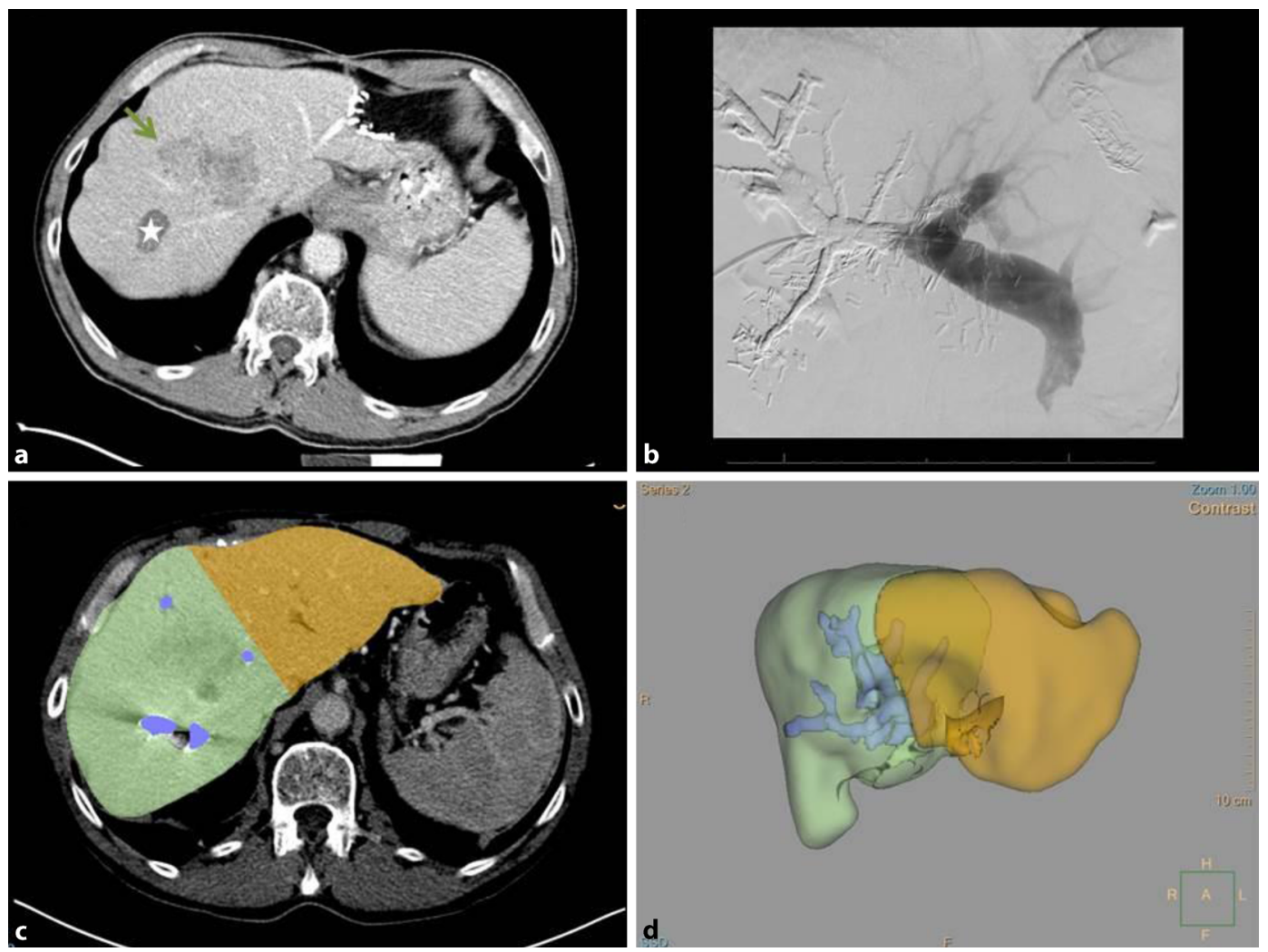

Abb. $2 \Delta$ CT-Bildgebung eines 66-jährigen männlichen Patienten mit bilobär synchron hepatisch metastasiertem Rektumkarzinom, UICC Stadium IV aus dem März 2015. Zur lokalen Kontrolle einer rechts subdiaphragmal (Segment VIII) gelegenen Metastase erfolgte eine perkutane Radiofrequenzablation (RFA). Nach Induktionschemotherapie erfolgte in 2-zeitiger Intention die Sanierung der Leber zunächst durch En-bloc-Resektion der Segmente IVa und V sowie Enukleation in Segment VI. 2-zeitige Sanierung der linken Leber synchron mit laparoskopischer tiefer anteriorer Rektumresektion (TAR) und totaler mesorektaler Exzision (TME). Nach adjuvanter Therapie zeigte sich in der ersten Nachsorge im März 2016 eine große Metastase im Segment V/VIII (a Metastase markiert mit grünem Pfeil; weißer Stern markiert die RFA-Narbe). Nach Reinduktion einer Systemtherapie erfolgte zur Hypertrophieinduktion der marginalen linken Restleber eine Embolisation des rechten Pfortaderastes (b). In der CT-Kontrolle erfolgte eine Lebervolumentrie (c,d) die eine Volumenzunahme des linken Leberlappens zeigt

Während die reine operative Ligatur der Pfortader bedingt durch das deutlich weniger invasive interventionelle Verfahren als alleiniges Verfahren nur noch sehr selten eingesetzt wird, sind weitere Methoden zur Induktion einer Hypertrophie von Lebergewebe 2- oder mehrzeitige Leberresektionen [15]. In den letzten Jahren wurde darüber hinaus eine neue vielversprechende Methode, insbesondere bei marginal resektablen Tumoren, aus Kombination von intraoperativer rechtsseitiger portalvenöser Ligatur und vollständiger Parenchymdissektion zur Hypertrophiedinduktion des linkslateralen Leberlappens bei primär funktionell irresektablen Lebermetasten eingeführt. Das sog. ALPPS-Verfahren kann sowohl alternative zur portalvenösen Embolisation, aber auch bei dessen Therapieversagern eingesetzt werden, allerdings mit signifikanter Morbidität und Mortalität (• Abb. 3; [16]).

\section{》) Die neoadjuvante \\ Chemotherapie zielt auf die Reduktion des Tumorvolumens}

Da im Verlauf nach primär kurativer Resektion etwa 60-70\% der Patienten ein Rezidiv entwickeln, das bei etwa einem Drittel erneut auf die Leber lokalisiert bleibt, sollten Reresektionen in Betracht gezogen werden. Sie sollte dann in Erwägung gezogen werden, wenn sich die Leber als einzige Rezidivlokalisation darstellt, das Rezidiv mit einer niedrigen Tumorlast einhergeht und die Tumorbiologie diesen Ansatz als sinnvoll erscheinen lässt. Morbididätsraten zwischen 11 und $52 \%$ und Mortalitätsraten von $1 \%$ stellen Reresektionen als sichere Therapieoptionen mit nur unwesentlichen Unterschieden zu primären Leberresektionen heraus. In der publizierten Literatur finden sich 3-Jahres-Überlensraten von über $60 \%$ und 5-Jahres-Überlebensraten von über $40 \%$ nach zweiter Resektion [17].

\section{Perioperative und adjuvante Chemotherapie}

Ziel einer neoadjuvanten perioperativen oder adjuvanten systemischen Chemotherapie ist es, die Ergebnisse einer rein chirurgischen Therapie $\mathrm{zu}$ verbessern. Vergleichende Analysen zwischen diesen Konzepten liegen derzeit nicht vor. In einer randomisierten Phase-III-Studie der European Organisation for Research and Treatment of Cancer (EORTC) unter- 

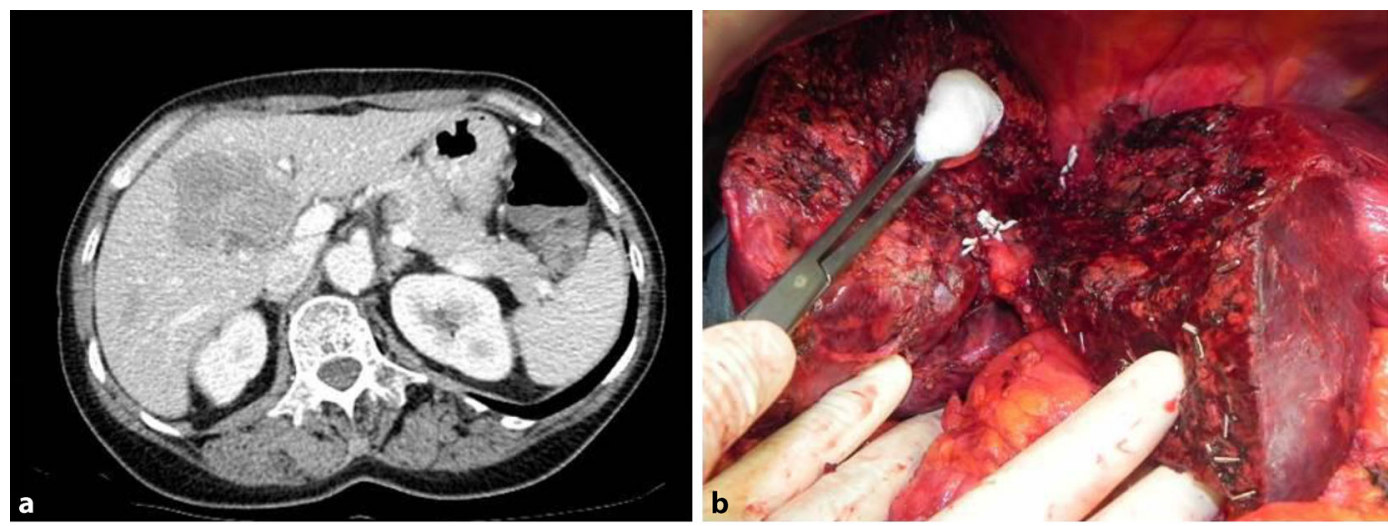

Abb. $3<$ CT-Bildgebung einer 64-jährigen Patienten mit metachron hepatisch metastasiertem kolorektalem Karzinom. Nach Induktionschemotherapie mit Folinsäure, 5-Fluorouracil und Oxaliplatin (FOLFOX) und Bevacizumab über 3 Monate mit partiellem Response erfolgte bei ungünstiger Lage der Metastase in Segment IV (a) sowie weiteren rechtshepatischen Metastasen
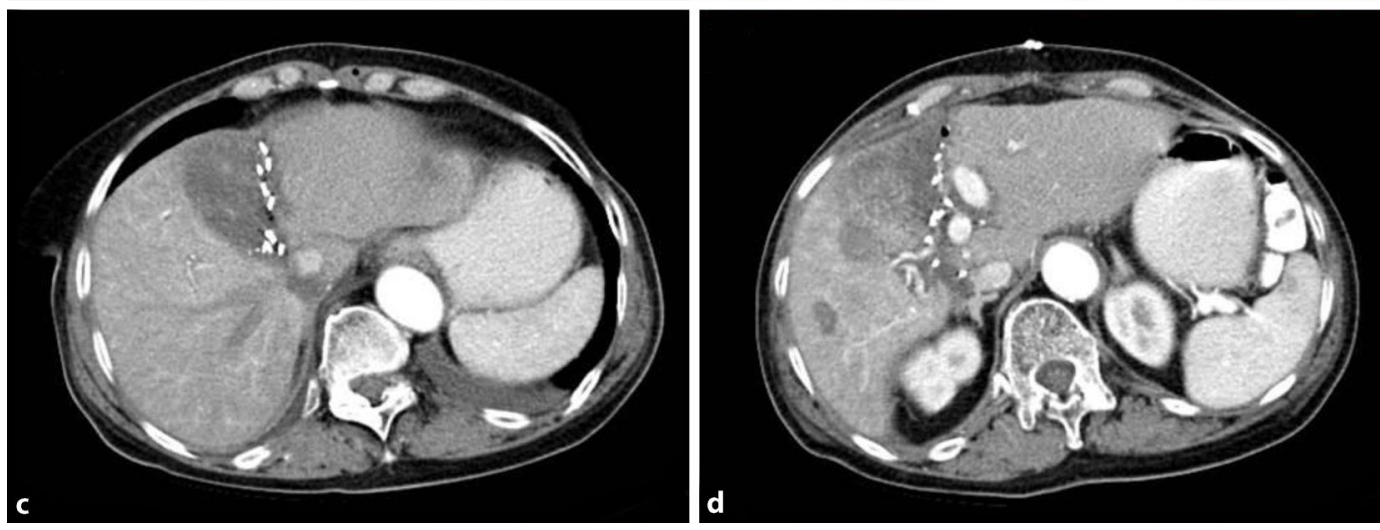
primär eine Trisektorektomie rechts (Segmente IVa, V, VI, VII und VIII) als „associating liver partition and portalvein ligation for staged hepatectomy" (ALPPS; b). Die Abbildungen c und $\mathbf{d}$ zeigen die Verlaufsbildgebung 7 Tage nach ALPPS mit erfolgter Parenchymdurchtrennung, aber noch in situ befindlichen rechtshepatischen Segmenten unmittelbar vor der zweiten Operation

suchte die Arbeitsgruppe von Nordlinger et al.den Stellenwert einer perioperativen Therapie mit Folinsäure, 5- Fluorouracil (5-FU) und Oxaliplatin (FOLFOX)4 bei Patienten mit primär als resektabel eingestuften Lebermetastasen eines kolorektalen Karzinoms (EPOC-Studie; [18]). Eingeschlossen wurden Patienten an 78 Zentren in Europa, Australien und Hongkong. Erlaubt waren bis zu 4 Lebermetastasen. Während die Intentionto-treat-Analyse hinsichtlich des krankheitsfreien 3-Jahres-Überlebens (PFS) keinen signifikanten Vorteil für die perioperative Therapie zeigte, ergab eine Perprotocol-Analyse, die nur die tatsächlich resezierten Patienten berücksichtigte, eine signifikante Verlängerung des PFS (etwa $8 \%$ ). Diese übertrug sich jedoch nicht in eine signifikante Verlängerung des Gesamtüberlebens, sodass die Studie nicht als uneingeschränkt positiv gewertet werden kann. Zu berücksichtigen ist die erhöhte perioperative Morbidität im Chemotherapiearm (25\% vs. $16 \%$ ), wobei die Gesamtmortalität nicht beeinflusst war [18].

Als potenzieller Vorteil einer neoadjuvanten/perioperativen Therapie wird die Möglichkeit einer frühen Behandlung von Mikrometastasen diskutiert. Eine Intensivierung der präoperativen Chemotherapie etwa durch Hinzunahme von Biologicals erschien daher als sinnvolle Weiterentwicklung dieses Konzepts. Die britische New-EPOC-Studie testete den Stellenwert des gegen den Rezeptor von „epidermal growth factor“ (EGFR) gerichteten Antikörpers Cetuximab in der perioperativen Therapie [19]. Eingeschlossen wurden $117 \mathrm{~Pa}$ tienten ohne Mutation in Exon 2 von KRAS. Nach einem medianen Followup von über 20 Monaten zeigte sich überraschend ein signifikant kürzeres PFS im Cetuximabarm (14,1 Monate vs. 20,5 Monate im Kontrollarm), sodass eine Intensivierung der perioperativen Therapie mit EGFR-Antikörpern ohne Zusatznutzen blieb. Wenngleich die Studie u. a. aufgrund einer weniger aggressiven Chirurgie im experimentellen Arm und weiteren Imbalancen zwischen den beiden Gruppen teilweise heftig kritisiert wurden, muss derzeit der Einsatz von Cetuximab in diesem Kontext kritisch gesehen werden. Randomisierte Daten zur Wirksamkeit von perioperativ verabreichtem Bevacizumab liegen nicht vor.

Vor dem Hintergrund der unsicheren Datenlage zur perioperativen Therapie wird insbesondere in den USA vielfach eine adjuvante Chemotherapie nach Resektion von Lebermetastasen eines kolorektalen Karzinoms empfohlen [20]. Grundlage hierfür sind indirekte Evidenzen aus den Studien zur adjuvanten Therapie von Patienten im nichtmetastasierten Stadium: Seit den 1990er-Jahren wurde in zahlreichen randomisierten Studien der Nutzen einer adjuvanten zytostatischen Therapie zur Senkung des Rückfallrisikos für Patienten im Stadium III bzw. Stadium II mit Risikofaktoren gezeigt $[21,22]$. Trotz der naheliegenden Gründe für den Versuch, das Wiederauftreten eines Tumors nach kurativer Resektion von Metastasen durch postoperative Chemotherapie zu verringern, sind die verfügbaren Daten für die metastasierte Situation allerdings spärlich. Die Wirksamkeit einer adjuvanten Chemotherapie bei Patienten mit metastasiertem KRK untersuchten 2 multizentrische PhaseIII-Studien, die einer klinisch vollständigen Metastasenresektion (auch Lungen- 


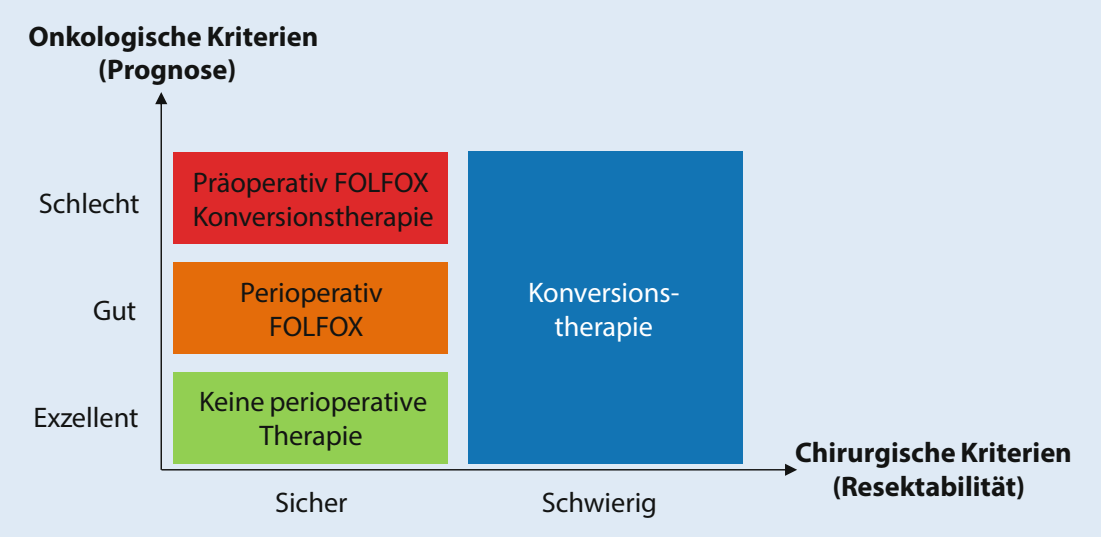

Abb. 4 ム Übersicht zur Behandlung von Patienten mit resektablen Lebermetastasen in Abhängigkeit onkologischer und chirurgischer Kriterien. FOLFOX Folinsäure, 5-Fluorouracil, Oxaliplatin. (Modifiziert nach [46])

metastasen erlaubt) unterzogen wurden (Francophone de Cancérologie Digestive [FFCD] 9002, EORTC/National Cancer Institute of Canada Clinical Trials Group/Gruppo Italiano di Valutazione Interventi in Oncologia [ENG]; [23, 24]). Beide ähnlich aufgestellten Studien zeigten eine grenzwertig signifikante Verbesserung des krankheitsfreien Überlebens, das Gesamtüberleben blieb jedoch unbeeinflusst. Aufgrund der sehr zögerlichen Rekrutierung wurden beide Studien vorzeitig geschlossen, sodass die statistische Power zum Nachweis von Unterschieden im Überleben nur unzureichend war. In beiden Studien wurde darüber hinaus ein nur wenig effektives und relativ toxisches Chemotherapieregime (5FU-Bolusapplikation) eingesetzt. In einer vielbeachteten Metaanalyse wurden beide Studien zusammenfassend analysiert [25]. Es waren insgesamt 278 Patienten auswertbar. Die mittlere Zeit bis zum Rezidiv (PFS) lag in der Chirurgiegruppe bei 18,8 Monaten und in der Gruppe der Patienten, die zusätzlich eine Chemotherapie erhielt, bei 27,9 Monaten. Das mediane Gesamtüberleben betrug in diesen Gruppen 47,3 bzw. 62,2 Monate. Die Gabe der Chemotherapie war in der Multivariatanalyse unabhängig mit dem PFS sowie dem Gesamtüberleben assoziiert.

Den möglichen Nutzen einer adjuvanten Chemotherapie nach Metastasenresektion zeigte schließlich auch eine retrospektive Registeranalyse, die 792 europäische und US-amerikanische Pati- enten einschloss [26]. Die Frage nach Intensivierung der Chemotherapie z. B. durch eine Hinzunahme von Oxaliplatin ist, anders als beim nichtmetastasierten Stadium, derzeit nicht durch Studien beantwortet, wird aber durch zahlreiche Autoren befürwortet. Antikörperbasierte Schemata sollten außerhalb von Studien nichtgegeben werden. Ebenso ist derStellenwert von lokal wirksamen Verfahren, wie der intraarteriellen Chemotherapie („hepatic arterial infusion chemotherapy") trotz einzelner positiver Studien [27, 28] unklar.

\section{\) Antikörperbasierte Schemata sollten außerhalb von Studien nicht gegeben werden}

In der klinischen Routine kommt bei Fehlen von klaren Evidenzen für eines der vorgestellten Konzepte ein abgestuftes Vorgehen in Abhängigkeit von onkologischen (prognostischen) und chirurgischen (technischen) Kriterien zur Anwendung: Patienten mit sicher resektabler Erkrankung und exzellenten prognostischen Faktoren benötigen keine neodadjuvante/perioperative Therapie und sollten unmittelbar reseziert werden (•Abb.4). Dementgegen können Patienten mit nicht exzellenten onkologischen oder chirurgischen Faktoren analog der Daten aus der EPOC-Studie einer perioperativen Kombinationschemotherapie zugeführt werden. Diese kann, unter Berücksichtigung von Vortherapien, in Höchstrisikosituationen oder beim Fehlen einer sicheren Resektabilität auch als „Konversionstherapi““, z. B. mit einem erweiterten Chemotherapie-Backbone oder mit Biologicals, durchgeführt werden. Patienten, die keine präoperative Chemotherapie erhalten haben, können abhängig von erfolgten Vortherapien und onkologischen Risikofaktoren einer adjuvanten Therapie zugeführt werden (• Abb. 4).

\section{Konversionstherapie}

Fortschritte in chirurgischen und intensivmedizinischen Techniken machen zunehmend auch ausgedehnte Leberbzw. Lungenresektionen möglich. Darüber hinaus bieten moderne Chemotherapiekombinationen die Möglichkeit einer teils deutlichen Tumorvolumenreduktion („tumor shrinkage“; • Abb. 4), sodass sekundäre Tumorresektionen zunehmend möglich werden. Patienten nach sekundärer Resektion weisen eine nur etwas schlechtere Prognose als Patienten nach primärer Resektion auf, aber eine bessere Prognose als auch sekundär nicht zu resezierende Patienten. Daher sollten alle Patienten mit einer limitierten, aber nicht primär resektablen Lebermetastasierung initial als Kandidaten für eine sekundäre Resektion betrachtet werden [29]. Die zunehmende Effektivität moderner Therapien macht frühe und regelmäßige Kontrollen der Resektabilität notwendig.

\section{》) Frühe und regelmäßige Kontrollen der Resektabilität sind notwendig}

Die Autoren empfehlen eine erste Evaluation spätestens 2 Monate nach Therapiebeginn und hiernach regelmäßig im Abstand von 2 Monaten für etwa $1 \mathrm{Jahr}$. Wenngleich eine maximale Tumorantwort erfahrungsgemäß nach 3-4 Monaten erreicht wird, werden viele Patienten erst zu einem späteren Zeitpunkt als resektabel eingeschätzt. In der sog. CELIMStudie wurde eine sekundäre Resektabilität spätestens 8 Monate nach Therapiebeginn erreicht [30]. 
Tab. 1 Ausgewählte aktuelle Studien der Arbeitsgemeinschaft für Internistische Onkologie (AIO) zur Therapie von Patienten mit metastasiertem Kolorektalkarzinom

\begin{tabular}{|c|c|c|c|}
\hline Studienname & Protokoll & Art & Besonderheit \\
\hline $\begin{array}{l}\text { AlO-KRK-0109 } \\
\text { (VOLFI) }\end{array}$ & FOLFOXIRI/Panitumumab vs. FOLFOXIRI & $1 \mathrm{~L}$ & $\begin{array}{l}\text { RAS-Wildtyp } \\
\text { Nichtresektabel }\end{array}$ \\
\hline $\begin{array}{l}\text { AIO-KRK-0114 } \\
\text { (FIRE-4) }\end{array}$ & $\begin{array}{l}\text { Cetuximabreexposition vs. Standardthe- } \\
\text { rapie nach wirksamer cetuximabbasierter } \\
\text { Erstlinienbehandlung }\end{array}$ & $1 / 3 \mathrm{~L}$ & $\begin{array}{l}\text { RAS-Wildtyp } \\
\text { Nichtresektabel }\end{array}$ \\
\hline $\begin{array}{l}\text { AAIO-KRK-0116 } \\
\text { (FIRE-4.5) }\end{array}$ & $\begin{array}{l}\text { FOLFOXIRI/Cetuximab vs. FOLFOXIRI/ } \\
\text { Bevacizumab }\end{array}$ & $1 \mathrm{~L}$ & $\begin{array}{l}\text { BRAF-mutiert } \\
\text { Nichtresektabel }\end{array}$ \\
\hline $\begin{array}{l}\text { AIO-KRK-0212 } \\
\text { (PanaMa) }\end{array}$ & $\begin{array}{l}\text { 5-FU/Panitumumab vs. 5-FU/FA nach Induk- } \\
\text { tion mit FOLFOX6/Panitumumab }\end{array}$ & M & RAS-Wildtyp \\
\hline $\begin{array}{l}\text { AIO-KRK-0313 } \\
\text { (IMPALA) }\end{array}$ & $\begin{array}{l}\text { Lefitolimod (MGN1703) vs. Standardtherapie } \\
\text { nach erfolgreicher Induktionstherapie }\end{array}$ & M & - \\
\hline (SYNCHRONOUS) & $\begin{array}{l}\text { Resektion des Primärtumors vs. keine Re- } \\
\text { sektion vor Beginn einer Chemotherapie bei } \\
\text { synchronen nichtresektablen Metastasen } \\
\text { eines KRK }\end{array}$ & $S$ & - \\
\hline $\begin{array}{l}\text { AIO-KRK-0314 } \\
\text { (PARLIM-Trial) }\end{array}$ & $\begin{array}{l}\text { Panitumumab vs. Kontrolle } \\
\text { nach Lebermetastasenresektion }\end{array}$ & A & RAS-Wildtyp \\
\hline $\begin{array}{l}\text { AIO-KRK-0115 } \\
\text { (CELIM3-Trial) }\end{array}$ & $\begin{array}{l}\text { Zusätzliche Metastasensektion vs. Chemo- } \\
\text { therapie ohne Resektion }\end{array}$ & $S$ & $\begin{array}{l}\text { Patienten mit } \\
\text { schlechter Prog- } \\
\text { nose }\end{array}$ \\
\hline (CELIM 2) & $\begin{array}{l}\text { FOLFOXIRI/Cetuximab vs. FOLFIRI/ } \\
\text { Cetuximab; FOLFOXIRI/Bevacizumab vs. } \\
\text { FOLFOXIRI }\end{array}$ & $\mathrm{N}$ & Nichtresektabel \\
\hline \multicolumn{4}{|c|}{$\begin{array}{l}1 L \text { Erstlinientherapie, } 3 L \text { Drittlinientherapie, } A \text { adjuvante Therapie, } M \text { Erhaltungstherapie, } N \text { neo- } \\
\text { adjuvante Therapie, S chirurgische Studie, FA Folinsäure; 5-FU 5-Fluorouracil; FOLFOX Folinsäure, } \\
\text { 5-Fluorouracil, Oxaliplatin, FOLFOXIRI FOLFOX plus Irinotecan; FOLFIRI Folinsäure, 5-Fluorouracil, } \\
\text { Irinotecan; KRK kolorektales Karzinom }\end{array}$} \\
\hline
\end{tabular}

Neben der Frage nach dem optimalen Kontrollzeitpunkt war lange Zeit auch die Wahl der „optimalen“ Konversionstherapie umstritten. Wichtigster Gesichtspunkt ist diesbezüglich, dass ein nahezu linearer Zusammenhang zwischen Tumoransprechen und sekundärer Resektabilität besteht, weshalb für Konversionstherapien insbesondere hochaktive Schemata („doublet/ triplet" plus Antikörper) infrage kommen. Die CELIM-Studie untersuchte bei Patienten mit technisch nichtresektablen Lebermetastasen den Stellenwert einer FOLFOX-Cetuximab-basierten Chemotherapie oder einer Therapie aus Folinsäure, 5-FU und Irinotecan (FOLFIRI) kombiniert mit Cetuximab [30]. Das Tumoransprechen lag bei $70 \%$ in der Gruppe der Patienten ohne Mutation in Exon 2 von KRAS. Es wurden $33 \%$ der Patienten sekundär reseziert, eine R0-Resektion war mit signifikant besserem Überleben assoziiert [31]. Eine Aussage zur Wirksamkeit von Cetuximab ermöglicht die Studie aufgrund des
In der nichtrandomisierten einarmigen BOXER-Studie wurde die Effektivität einer neoadjuvanten Gabe von Bevacizumab in Kombination mit Capecitabin und Oxaliplatin (CAPOX) bei initial nichtresektablen Patienten untersucht. Es zeigte sich eine bemerkenswerte Ansprechrate von $78 \%$, auch die R0Resektionsrate war überdurchschnittlich hoch [33]. Bevacizumabassoziierte Grad3-bis-4-Toxizitäten waren nicht zu verzeichnen. Eine weitere Intensivierung der Therapie wurde im Rahmen der europäischen OLIVIA-Studie getestet [34]. Patienten mit initial nichtresektablen Lebermetastasen wurden zwischen FOLFOX plus Irinotecan (FOLFOXIRI) bzw. FOLFOX jeweils kombiniert mit Bevacizumab randomisiert. Die Therapieintensivierung führte $\mathrm{zu}$ einer Steigerung des Tumoransprechens auf $81 \%$ in der FOLFOXIRI-Gruppe und damit einhergehend hohen Resektionsraten von $61 \%$. Die französische Methep-2-Studie verglich in einer ähnlichen Patientengruppe die Gabe von FOLFIRI plus Oxaliplatin (FOLFIRINOX) und Bevacizumab mit einer Standardtherapie aus FOLFOX oder FOLFIRI plus Bevacizumab [35]. Es zeigte sich eine deutliche Besserung aller Endpunkte durch die Eskalation des Chemotherapie-Backbones. Wenngleich der Anteil von Bevacizumab aufgrund des Fehlens eines entsprechenden Kontrollarms unklar bleibt, weisen die Daten eindrucksvoll auf Chancen einer intensivierten Chemotherapie bei Patienten mit initial nichtresektablen Lebermetastasen hin. Dem erhöhten Ansprechen steht allerdings eine signifikante Erhöhung der Toxizität entgegen, was eine aufmerksame Selektion und Überwachung der Patienten notwendig macht. Die Autoren beschränken die Gabe von FOLFOXIRI auf Patienten $<70$ Jahre in gutem Allgemeinzustand (Eastern Cooperative of Oncology Group [ECOG] 0-1) ohne wesentliche Einschränkung der Organfunktion.

In der klinischen Routine sollte zum Erreichen einer sekundären Resektabilität die Konversionstherapie mit möglichst effektiven Kombinationen durchgeführt werden. In RAS-mutierten $\mathrm{Pa}$ tienten wird die Therapie in der Regel als Doublette mit Bevacizumab geführt, 
alternativ kann bei ausgewählten Patienten FOLFOXIRI mit oder ohne Bevacizumab erwogen werden. Bei Patienten ohne RAS-Mutation wird eine EGFR-basierte Therapie in vielen Zentren bevorzugt. Unter Berücksichtigung einer retrospektiven Auswertung der deutschen FIRE-3Studie oder der amerikanischen CALGB/ SWOG-80.405-Studie, die eine verminderte Wirkung von Cetuximab bei Patienten mit rechtsseitigen Primarius nahelegen $[36,37]$, sollten aus Sicht der Autoren derzeit nur Patienten mit Tumoren distal der rechten Flexur mit EGFR-Antikörpern (Cetuximab, Panitumumab) behandelt werden. In Patienten mit einem rechtsseitigen Primarius kann FOLFOXIRI mit/ohne Bevacizumab oder auch eine Doublettentherapie mit Bevacizumab erwogen werden. Ein besonderes Kollektiv stellen Patienten mit BRAFV600E-Mutation dar. Basierend auf den Daten der TRIBE-Studie kann für diese Patienten FOLFOXIRI mit/ohne Bevacizumab erwogen werden, falls keine Kontraindikationen bestehen und sofern ein Studieneinschluss nicht möglich ist.

\section{Lokaltherapien}

Die meisten Erfahrungen zur lokal-ablativen Therapie bei Lebermetastasen eines kolorektalen Karzinoms liegen für die RFA vor [29]. Hierbei wird über eine Sonde, an die ein hochfrequenter Wechselstrom angelegt wird, Energie in das Gewebe eingebracht, wodurch es zur Hitzeentwicklung kommt. Aufgrund physikalischer Gegebenheiten ist das potenziell zu abladierende Areal auf einen Durchmesser von $4-5 \mathrm{~cm}$ limitiert. In der Nachbarschaft von größeren Blutgefäßen ist die Effektivität nochmals limitiert [29, 38]. Eine kürzlich veröffentlichte Analyse zur Lokalrezidivrate im Langzeitverlauf zeigte bei über 200 behandelten Patienten eine Lokalrezidivrate von $34 \%$ [39]. Trotz großer Fortschritte in den lokal-ablativen Verfahren kann insbesondere in derkurativen Situation keines der Verfahren vergleichbare Ergebnisse im Hinblick auf Überlebens- und Rezidivraten wie die chirurgische Resektion erzielen. Kritisch anzumerken ist, dass prospektive randomisierte Analysen diesbezüglich weitgehend fehlen. Die meisten Autoren sehen daher die Indikation eher ergänzend zur chirurgischen Therapie, insbesondere bei Patienten, bei denen eine komplette Tumorresektion nicht möglich ist, die aber durch ergänzende Ablation in eine R0Situation gebracht werden können [40, 41].

Häufig wird die Kombinationen aus ablativen Verfahren und Chirurgie bei Patienten genutzt, bei denen ein bilobärer Befall der Leber vorliegt. Hier werden durch die Resektion ein Großteil der Tumorlast entfernt und die nicht zu resezierenden Metastasen lokal-ablativ behandelt. Als Weiterentwicklung der RFA steht an einigen Zentren die noch experimentelle irreversible Elektroporation (IRE) zur Verfügung. Es handelt sich um ein nichtthermisches Verfahren, das durch kurzwellige Elektroimpulse die irreversible Zellmembranpolarisierung und somit die Zellapoptose im Ablationsareal induziert [40].

\section{》) Bei bilobärem Leberbefall wird die Kombinationen aus ablativen Verfahren und Chirurgie genutzt}

Das Indikationsspektrum lokal- und regional-ablativer Verfahren hat sich in den letzten Jahren grundsätzlich gewandelt: Nachdem längere Zeit lokal-ablative Verfahren alleine oder ergänzend zur Chirurgie in kurativen Konzepten zur Anwendung gebracht wurden, haben diese Verfahren mittlerweile einen durch prospektive Studien gesicherten Wert im Rahmen multimodaler palliativer Konzepte. Die CLOCC-Studie testete den Stellenwert einer RFA ergänzend zur Chemotherapie bei Patienten mit metastasiertem kolorektalem Karzinom in palliativer Intention [42, 43]. Eingeschlossen wurden Patienten mit bis zu 9 Lebermetastasen ohne extrahepatischem Befall, die als initial nichtresektabel angesehen wurden. Der Chemotherapieteil bestand aus FOLFOX, ein Teil der Patienten erhielt auBerdem Bevacizumab. Falls im Rahmen der Therapie eine chirurgische Resektion möglich wurde, durfte diese durchgeführt werden. Die Kombination aus Chemotherapie und RFA ergab ein signifikant erhöhtes mittleres Überleben (45,6 Monate) gegenüber dem Chemotherapiearm der Studie (40,5 Monate). Vor allem erbrachte die Studie mit einem 8-JahresGesamtüberleben von $35,9 \%$ ein beachtenswertes Langzeitergebnis für die mit RFA, Chemotherapie und ggf. chirurgischer Resektion behandelten Patienten gegenüber dem Arm ohne RFA (8,9\%). Dies unterstreicht eindrucksvoll das Potenzial einer multimodalen Therapie für initial nichtresektable Patienten mit kolorektalen Lebermetastasen. Im Rahmen der SIRFLOX-Studie erhielten chemotherapienaive Patienten mit metastasiertem Kolorektalkarzinom entweder FOLFOX (mit oder ohne Bevacizumab) oder FOLFOX plus SIRT (mit oder ohne Bevacizumab). Etwa $40 \%$ der eingeschlossenen Patienten wiesen einen extrahepatischen Befall auf. Erwartungsgemäß beeinflusste die SIRT lediglich den hepatischen Progress der Erkrankung, das PFS blieb unbeeinflusst [45]. Weitere Studien $(\mathrm{EPOCH})$ testen den Einsatz der SIRT in der Zweitlinientherapie. Derzeit kann die SIRT außerhalb von Studien allenfalls nach Versagen konventioneller Therapien mit dem Ziel einer hepatischen Tumorkontrolle empfohlen werden.

\section{Zukünftige Entwicklungen und aktuelle Studien}

Trotz wichtiger Fortschritte in der interdisziplinären Therapie von Patienten mit KLM sind weiterhin viele wichtige Fragen offen, von denen einige in aktuellen klinischen Studien untersucht werden. So ist weiterhin unklar, ob Patienten mit primär resektablen KLM von einer perioperativen oder adjuvanten Systemtherapie profitieren und wie genau diese durchgeführt werden sollte. Aktuell wird in der PARLIM-Studie die zusätzliche Gabe von Panitumumab zu FOLFOX in der adjuvanten Therapie nach Metastasenresektion bei RASWildtyp-Patienten untersucht (• Tab. 1; EudraCT-Nr.: 2010-023671-26). Eine weitere wichtige Frage betrifft die optimale Chemotherapie zur Konversion bei initial nichtresektablen Lebermetastasen. In der deutschen CELIM2-Studie wird in einem 4-armigen Design untersucht, ob bei nichtresektablen Patienten 
mit hepatisch limitierter Metastasierung eine Intensivierung der Chemotherapie (RAS-Wildtyp: Cetuximab/FOLFOXIRI vs. Cetuximab/FOLFIRI; RAS-mutiert: FOLFOXIRI/Bevavizumab vs. FOLFOXIRI) ein verbessertes Ansprechen sowie eine erhöhte Rate an R0-Resektionen bewirkt. Weiterhin wird ein zunehmender Schwerpunkt auf der Frage nach neuen Biomarkern liegen, die ein mögliches Therapieansprechen und eine zu erwartende Toxizität vorhersagen können und damit eine bessere Zuordnung der $\mathrm{Pa}$ tienten $\mathrm{zu}$ bestimmten Therapieformen ermöglichen.

Da zurzeit mehr Fragen als Antworten in diesem Bereich bestehen, ist die Entscheidung bez. der adäquaten Therapie immer individuell an den Patienten und seine Bedürfnisse anzupassen und kann nur sinnvoll in einer interdisziplinären Tumorkonferenz mit Einbeziehung aller beteiligten Fachdisziplinen getroffen werden. Da in eine solche Entscheidung immer auch die jeweiligen Erfahrungen und gewisse Präferenzen an einzelnen Zentren Einfluss haben werden, ist gegebenenfalls das Einholen einer Zweitmeinung für den Patienten sinnvoll und in Deutschland für onkologische Patienten zunehmend gängige Praxis [44]. Schließlich ist eine gute Kooperation und Kommunikation zwischen dem niedergelassenen Behandler und einem Darmkrebszentrum am Krankenhaus essenziell, um gemeinsam dem Patienten eine erfolgreiche, multimodale Therapie anbieten $\mathrm{zu}$ können, als deren wichtiger Teil auch der Einschluss in eine klinische Studie (- Tab. 1) erwogen werden sollte.

\section{Fazit für die Praxis}

Durch den Einsatz multimodaler
Therapiekonzepte sind auch im
metastasierten Stadium ein Lang-
zeitverlauf und sogar eine Heilung
möglich.
Patienten mit einem kolorektalen
Karzinom sollten zu Beginn der Be-
handlung in einer interdisziplinären
Tumorkonferenz und unter Einbezug
eines in der Leberchirurgie erfahre-
nen Chirurgen besprochen werden.
Resektablen Lebermetastasen sollten
reseziert werden. Eine neoadjuvante,

adjuvante oder perioperative Systemtherapie kann gegebenenfalls erwogen werden.

- Bei primär irresektablen Lebermetastasen sollte, falls eine sekundäre Resektion möglich scheint, eine Konversionstherapie erfolgen. Sekundärer resektable Tumoren sollten entfernt werden.

- Auch im Verlauf einer längerfristigen Therapie ist im Rahmen einer Konferenz regelmäßig zu prüfen, ob alternative Therapieformen sinnvoll sind.

- Lokal- und regional-ablative Verfahren können bei ausgewählten Patienten mit Chemotherapien und ggf. chirurgischen Resektionen bereits in frühen Linien kombiniert werden.

\section{Korrespondenzadresse

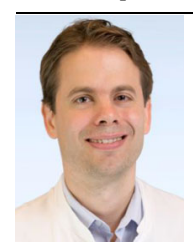 \\ Univ.-Prof. Dr. T. Luedde, PhD \\ Mildred-Scheel-Professor für Gastroenterologie, Hepatologie und hepatobiliäre Onkologie, Universitätsklinikum RWTH Aachen \\ Aachen, Deutschland tluedde@ukaachen.de}

Danksagung. Die Autoren danken PD Dr. S. Kasper für die kritische Durchsicht des Manuskripts.

\section{Einhaltung ethischer Richtlinien}

Interessenkonflikt. T. Luedde, C. Roderburg, M. Binnebösel, P. Neumann und C. Trautwein erhielten in der Vergangenheit Vortragshonorare und/oder Reiseunterstützung und/oder waren als Prüfarzt im Rahmen klinischer Studien tätig, in denen Substanzen der folgenden Firmen untersucht wurden: Amgen $\mathrm{GmbH}$, Bayer AG, Bristol-Meyer Squibb GmbH \& Co, Merck $\mathrm{KGaA}$, Roche Pharma AG, Sanofi-Aventis Deutschland $\mathrm{GmbH}$.

Dieser Beitrag beinhaltet keine von den Autoren durchgeführten Studien an Menschen oder Tieren.

Open Access. Dieser Artikel wird unter der Creative Commons Namensnennung 4.0 International Lizenz (http://creativecommons.org/licenses/by/4.0/deed. de) veröffentlicht, welche die Nutzung, Vervielfältigung, Bearbeitung, Verbreitung und Wiedergabe in jeglichem Medium und Format erlaubt, sofern Sie den/die ursprünglichen Autor(en) und die Quelle ordnungsgemäßnennen, einen Link zur Creative Commons Lizenz beifügen und angeben, ob Änderungen vorgenommen wurden.

\section{Literatur}

1. Zentrum für Krebsregisterdaten des RKI. http://www.krebsdaten.de/Krebs/DE/Content/ Krebsarten/Darmkrebs/darmkrebs_node.html. Zugegriffen:01.08.2016

2. Abdalla EK, Adam R, Bilchik AJ, Jaeck D, Vauthey JN, Mahvi D (2006) Improving resectability of hepatic colorectal metastases: Expert consensus statement. Ann Surg Oncol 13(10):1271-1280

3. Donadon M, Ribero D, Morris-Stiff G, Abdalla EK, Vauthey JN (2007) New paradigm in the management of liver-only metastases from colorectal cancer. Gastrointest Cancer Res 1(1):20-27

4. AbbasS, LamV,Hollands M(2011)Ten-yearsurvival after liver resection for colorectal metastases: Systematic review and meta-analysis. Isrn Oncol. doi:10.5402/2011/763245

5. Pox C, Aretz S, Bischoff SC, Graeven U, Hass M, Heussner P et al (2013) S3-guideline colorectal cancer version 1.0.Z Gastroenterol 51(8):753-854

6. Jara M, Reese T, Malinowski M, Valle E, Seehofer D, Puhl $G$ et al (2015) Reductions in posthepatectomy liver failure and related mortality after implementation of the LiMAx algorithm in preoperative work-up: A single-centre analysis of 1170 hepatectomies of one or more segments. HPB 17(7):651-658

7. Angelsen JH, Horn A, Eide GE, Viste A (2014) Surgery for colorectal liver metastases: The impact of resection margins on recurrence and overall survival. World J Surg Oncol 12(1):127. doi:10. 1186/1477-7819-12-127

8. Fong Y, Cohen AM, Fortner JG, Enker WE, Turnbull AD, CoitDGetal (1997) Liver resection for colorectal metastases. JClin Oncol 15(3):938-946

9. Buchs NC, Ris F, Majno PE, Andres A, Cacheux W, Gervaz P et al (2015) Rectal outcomes after a liverfirst treatment of patients with stage IV rectal cancer. Ann Surg Oncol 22(3):931-937

10. Tamandl D, Klinger M, Eipeldauer S, Herberger B, Kaczirek K, Gruenberger B et al (2011) Sinusoidal obstruction syndrome impairs long-term outcome of colorectal liver metastases treated with resection after neoadjuvant chemotherapy. Ann Surg Oncol 18(2):421-430

11. Pessaux $P$, Chenard MP, Bachellier $P$, Jaeck D (2010) Consequences of chemotherapy on resection of colorectal liver metastases. J Visc Surg 147(4):e193-201

12. Wieser $M$, Sauerland $S$, Arnold D, Schmiegel W, Reinacher-Schick A (2010) Peri-operative chemotherapy for the treatment of resectable liver metastases from colorectal cancer: A systematic review and meta-analysis of randomized trials. BMC Cancer. doi:10.1186/1471-2407-10-309

13. Schmoll HJ, Van Cutsem E, Stein A, Valentini V Glimelius B, Haustermans K et al (2012) ESMO Consensus Guidelines for management of patients with colon and rectal cancer. A personalized approach to clinical decision making. Ann Oncol 23(10):2479-2516

14. van Lienden KP, van den Esschert JW, de Graaf W, Bipat S, Lameris JS, van Gulik TM et al (2013) Portal vein embolization before liver resection: A systematic review. Cardiovasc Intervent Radiol 36(1):25-34

15. Adam R, Miller R, Pitombo $M$, Wicherts DA, de Haas RJ, Bitsakou G et al (2007) Two-stage hepatectomy approach for initially unresectable colorectal hepatic metastases. Surg Oncol Clin N Am 16(3):525-536

16. Hasselgren K, Sandstrom P, Bjornsson B (2015) Role of associating liver partition and portal vein 
ligation for staged hepatectomy in colorectal liver metastases: A review. World J Gastroenterol 21(15):4491-4498

17. Petrowsky H, Gonen M, Jarnagin W, Lorenz M, DeMatteo R, Heinrich $S$ et al (2002) Second liver resections are safe and effective treatment for recurrent hepatic metastases from colorectal cancer: A bi-institutional analysis. Ann Surg 235(6):863-871

18. Nordlinger B, Sorbye H, Glimelius B, Poston GJ, Schlag PM, Rougier P et al (2013) Perioperative FOLFOX4 chemotherapy and surgery versus surgery alone for resectable liver metastases from colorectal cancer (EORTC 40983): Long-term results of a randomised, controlled, phase 3 trial. Lancet Oncol 14(12):1208-1215

19. Primrose J, FalkS, Finch-Jones M, Valle J, O’Reilly D, Siriwardena A et al (2014) Systemic chemotherapy with or without cetuximab in patients with resectable colorectal liver metastasis: The New EPOC randomised controlled trial. Lancet Oncol 15(6):601-611

20. Benson AB 3rd, Venook AP, Bekaii-Saab T, Chan E, Chen YJ, Cooper HS et al (2014) Colon cancer, version 3.2014. J Natl Compr Cancer Netw 12(7):1028-1059

21. Andre T, Boni C, Navarro M, Tabernero J, Hickish T, Topham C et al (2009) Improved overall survival with oxaliplatin, fluorouracil, and leucovorin as adjuvant treatment in stage II or III colon cancer in the MOSAIC trial.J Clin Oncol 27(19):3109-3116

22. Schmoll HJ, Twelves $C$, Sun W, O'Connell MJ, Cartwright T, McKenna E et al (2014) Effect of adjuvant capecitabine or fluorouracil, with or without oxaliplatin, on survival outcomes in stage III colon cancer and the effect of oxaliplatin on post-relapse survival: A pooled analysis of individual patient data from four randomised controlled trials. Lancet Oncol 15(13):1481-1492 (PubMed PMID: 25456367. Pubmed Central PMCID: 4435787)

23. Portier G, Elias D, Bouche O, Rougier P, Bosset JF, Saric J et al (2006) Multicenter randomized trial of adjuvant fluorouracil and folinic acid compared with surgery alone after resection of colorectal liver metastases: FFCD ACHBTH AURC 9002 trial. JClin Oncol 24(31):4976-4982

24. Langer B, Bleiberg H, Labianca R, Shepherd L (2002) Fluorouracil (FU) plus I-leucovorin (I-LV) versus observation after potentially curative resection of liver or lung metastases from colorectal cancer (CRC): Results of the ENG (EORTC/NCIC CTG/GIVIO) randomized trial. Proc Am Soc Clin Oncol 21:592

25. Mitry E, Fields AL, Bleiberg H, Labianca R, Portier G, Tu D et al (2008) Adjuvant chemotherapy after potentially curative resection of metastases from colorectal cancer: A pooled analysis of two randomized trials. JClin Oncol 20;26(30):4906-4911

26. Parks R, Gonen M, Kemeny N, Jarnagin W, D'Angelica M, DeMatteo R et al (2007) Adjuvant chemotherapy improves survival after resection of hepatic colorectal metastases: Analysis of data from two continents. J Am Coll Surg 204(5):753-761

27. Alberts SR, Roh MS, Mahoney MR, O'Connell MJ, Nagorney DM, Wagman L et al (2010) Alternating systemic and hepatic artery infusion therapy for resected liver metastases from colorectal cancer: A North Central Cancer Treatment Group (NCCTG)/ National Surgical Adjuvant Breast and Bowel Project (NSABP) phase ll intergroup trial, N9945/Cl66. JClin Oncol 28(5):853-858
28. Kemeny NE, Gonen M (2005) Hepatic arterial infusion after liver resection. $\mathrm{N}$ Engl J Med 352(7):734-735

29. Tomlinson JS, Jarnagin WR, DeMatteo RP, Fong Y, Kornprat P, Gonen M et al (2007) Actual 10-year survival after resection of colorectal liver metastases defines cure. J Clin Oncol 25(29):4575-4580

30. Folprecht $G$, Gruenberger T, Bechstein WO, Raab HR, Lordick F, Hartmann JT et al (2010) Tumour response and secondary resectability of colorecta liver metastases following neoadjuvant chemotherapy with cetuximab: the CELIM randomised phase 2 trial. Lancet Oncol 11(1):38-47

31. Folprecht G, Gruenberger T, Bechstein W, Raab HR, Weitz J, Lordick F et al (2014) Survival of patients with initially unresectable colorectal liver metastases treated with FOLFOX/cetuximab or FOLFIRI/cetuximab in a multidisciplinary concept (CELIM study). Ann Oncol 25(5):1018-1025

32. Ye LC, Liu TS, Ren L, Wei Y, Zhu DX, Zai SY etal (2013) Randomized controlled trial of cetuximab plus chemotherapy for patients with KRAS wild-type unresectable colorectal liver-limited metastases. J Clin Oncol 31(16):1931-1938

33. Wong R, Cunningham D, Barbachano Y, Saffery $C$, Valle J, Hickish T et al (2011) A multicentre study of capecitabine, oxaliplatin plus bevacizumab as perioperative treatment of patients with poor-risk colorectal liver-only metastases not selected for upfront resection. Ann Oncol 22(9):2042-2048

34. Gruenberger T, Bridgewater J, Chau I, Garcia Alfonso P, Rivoire M, Mudan S et al (2015) Bevacizumab plus mFOLFOX-6 or FOLFOXIR in patients with initially unresectable liver metastases from colorectal cancer: The OLIVIA multinational randomised phase II trial. Ann Oncol 26(4):702-708

35. Ychou M, Rivoire M, Thezenas S, Guimbaud R, Ghiringhelli F, Mercier-Blas A, Mineur L, Francois E, Khemissa F, Moussata D, Becouarn Y, Houyau P Aparicio T, Adam R, Galais MP, Audemar F, Assenat E, Stanbury T, Bouche O (2016) FOLFIRINOX combined to targeted therapy according RAS status for colorectal cancer patients with liver metastases initially non-resectable: A phase II randomized Study - Prodige 14 - ACCORD 21 (METHEP-2), a unicancer GI trial. J Clin Oncol 34(suppl):abstr 3512

36. Venook AP, Niedzwiecki D, Innocenti F, Fruth $B$, Greene C, O'Neil BH, Shaw JE, Atkins JN, Horvath LE, Polite BN, Meyerhardt JA, O'Reilly EM, Goldberg RM, Hochster HS, Blanke CD, Schilsky RL, Mayer RJ, Bertagnolli MM, Lenz HJ (2016) Impact of primary (10) tumor location on overall survival (OS) and progression-free survival (PFS) in patients (pts) with metastatic colorectal cancer ( $\mathrm{mCRC}$ ): Analysis of CALGB/SWOG 80405 (Alliance). J Clin Oncol 34(suppl):abstr 3504

37. Heinemann V, Modest DP, von Weikersthal LF, Decker T, Kiani A, Vehling-Kaiser U, Al-Batran SE Heintges T, Lerchenmuller CA, Kahl C, Seipelt G, Kullmann F, Stauch M, Scheithauer W, Held $S$, Giessen CA, Jung A, Kirchner T, Stintzing $S$ (2014) Gender and tumor location as predictors for efficacy: Influence on endpoints in first-line treatment with FOLFIRI in combination with cetuximab or bevacizumab in the AIO KRK 0306 (FIRE3) trial. J Clin Oncol 32(5s suppl):abstr 3600

38. Wright AS, Sampson LA, Warner TF, Mahvi DM, LeeFT Jr. (2005) Radiofrequency versus microwave ablation in a hepatic porcine model. Radiology 236(1):132-139
39. Amersi FF, McElrath-Garza A, Ahmad A, Zogaki T, Allegra DP, Krasne $R$ et al (2006) Longterm survival after radiofrequency ablation of complex unresectable liver tumors. Arch Surg 141(6):581-587 (discussion 7-8)

40. Binnebosel M, Bruners P, KlinkCD, KuhlC, Neumann UP (2016) Oligometastasized stage IV colorectal cancer: Surgical resection and local ablative procedures. Chirurg 87(5):371-379

41. Cirocchi R, Trastulli S, Boselli C, Montedori A, Cavaliere D, Parisi A et al (2012) Radiofrequency ablation in the treatment of liver metastases from colorectal cancer. Cochrane Database Syst Rev 6:CD006317

42. Tanis $E$, Nordlinger $B$, Mauer $M$, Sorbye $H$, van Coevorden F, Gruenberger T et al (2014) Local recurrence rates after radiofrequency ablation or resection of colorectal liver metastases. Analysis of the European Organisation for Research and Treatment of Cancer \#40004 and \#40983. Eur J Cancer 50(5):912-919

43. Ruers T, Punt $C$, van Coevorden F, Pierie JP, Rinkes IB, Ledermann JA, Poston GJ, Bechstein WO, Lentz MA, Mauer ME, Van Cutsem E, Lutz MP, Nordlinger $B$ (2015) Radiofrequency ablation (RFA) combined with chemotherapy for unresectable colorectal liver metastases (CRC LM): Long-term survival results of a randomized phase II study of the EORTC-NCRI CCSG-ALM Intergroup 40004 (CLOCC). JClin Oncol 33(suppl):abstr 3501

44. Ruetters D, Keinki C, Schroth S, Liebl P, Huebner $J$ (2016) Is there evidence for a better health care for cancer patients after a second opinion? A systematic review. J Cancer Res Clin Oncol 142(7):1521-1528

45. van Hazel GA, Heinemann V, Sharma NK, Findlay M, Ricke J, Peeters M, Perez D (2016) SIRFLOX: Randomized phase III trial comparing first-line mFOLFOX6 (plus or minus Bevacizumab) versus mFOLFOX6 (plus or minus Bevacizumab) plus selective internal radiation therapy in patients with metastatic colorectal cancer. J Clin Oncol 20:1723-1731

46. Van Cutsem E, Cervantes A, Adam R, Sobrero A, Van Krieken JH, Aderka D, Aranda Aguilar E et al (2016) ESMO consensus guidelines for the management of patients with metastatic colorectal cancer. Ann Oncol 27:1386-1422 